\title{
Los centros financieros regionales en Asia-Pacífico: Hong-Kong
}

$\mathrm{E}$ 1 creciente papel que han venido desempeñando los países asiáticos en la economía mundial desde los años sesenta ha ido acompañado de un incremento simultáneo de su participación en los flujos financieros globales y de un desarrollo sostenido de las plazas financieras de la región.

Los centros financieros internacionales (CFIs) constituyen un componente clave de la economía global, y sus funciones van más allá de servir de intermediarios entre ahorro e inversión, dado que son también centros de comunicación y de gestión global, insertos en densas redes que los conectan (Reed, 1981, en Goldberg, 1996). Los CFIs dominantes a nivel global, encabezados por las plazas de Nueva York, Londres, Ámsterdam y Zurich, se encuentran ubicados en economías desarrolladas que constituyen, o constituyeron (en los casos de Gran Bretaña y de los Países Bajos), el corazón del sistema capitalista mundial, y/o cuyas divisas ostentan el privilegio de ser monedas de reserva a nivel internacional. Su surgimiento se relacionó con la existencia de un hinterland industrial densamente poblado de empresas con intereses internacionales, y con una fuerte base de ahorro interno cuyos excedentes se canalizaban hacia inversiones externas (Lee, 1986; Montes, 1999). La evolución de la plaza de Tokio hacia su transformación en un CFI en la década de 1980 siguió un patrón similar: se fundamentó en la posición adquirida por este país en la economía mundial, en la abundancia de su ahorro interno y en la revaloración del yen (Montes, 1999).

Ahora bien, Asia-Pacífico cuenta hoy en día con una verdadera red de CFIs establecidos o emergentes: entre los primeros destacan las plazas de Hong Kong, Singapur y Sydney

* Investigadora del Departamento de Estudios del Pacífico de la Universidad de Guadalajara.
(Australia); entre los segundos, figuran plazas cuya importancia relativa y grados de internacionalización divergen ampliamente. Taipei (Taiwan), la región de Osaka en Japón, Manila (Filipinas), Bangkok (Tailandia), Kuala Lumpur, la plaza internacional offshore de Labuan (Malasia) y Shanghai (China continental) son mencionadas con mayor frecuencia (Goldberg, 1996; Law, 1999; Lee, 1986; Montes, 1999). El hecho de que estas plazas, con las excepciones de Osaka y Sydney, estén ubicadas en economías de desarrollo tardío, sugiere que su constitución como CFI no siguió al patrón de las plazas dominantes sino que obedeció a otras fuerzas.

De hecho, el desarrollo de estos CFIs está estrechamente vinculado con el proceso de globalización financiera; el surgimiento de las primeras plazas financieras regionales está asociado a la etapa inicial de este proceso, centrada alrededor de la expansión internacional del euromercado de divisas ${ }^{1}$ mientras la posterior multiplicación de los CFIs emergentes se da en la última etapa de desreglamentación y de constitución de mercados financieros globales, combinándose con la voluntad de los gobiernos de atraer la industria de los servicios financieros y captar parte de su actividad global ${ }^{2}$.

Este texto se propone analizar de manera comparativa el proceso formativo, las principales instituciones y el papel desempeñado por dos CFIs sobresalientes de la región, Hong Kong y Singapur, y establecer los retos a los cuales se enfrentan actualmente. Existen puntos en común y diferencias en el recorrido de ambas plazas hacia la actividad de CFI: en ambos casos se trata de ciudades que cuentan con recursos y con un territorio reducido, donde predomina la población de origen étnico chino; ambas llegaron a desempeñar históricamente, desde el período 
colonial, el papel deentrepôt comercial para el tránsito y la reexportación de productos de los países o territorios circundantes y fungieron luego como bases regionales de firmas multinacionales. En ambos casos también, el surgimiento de la actividad financiera se inició en la década de 1970 con la internacionalización del euromercado, y en un marco de plena libertad de flujos de capitales. Ahora bien, una diferencia fundamental entre Hong Kong y Singapur reside ciertamente en las políticas aplicadas para lograr su transformación en CFI. En el primer caso, se trató de políticas iniciales delaissezfaire, asociadas, más adelante, con un paulatino proceso de reforzamiento de la reglamentación; este patrón de desarrollo contrasta agudamente con el extenso fomento público aplicado en el segundo caso.

El presente texto es la primera parte de un artículo que consta de dos partes y seis secciones y tratará esencialmente de Hong Kong. La primera sección se inicia con una breve revisión de literatura aplicada a los CFIs. En las secciones segunda y tercera se revisa respectivamente el desarrollo y las principales instituciones (bancos y mercados de valores) del CFI de Hong Kong. La segunda parte, que se publicará en el próximo número de esta revista, abarca las secciones cuarta y quinta, donde se revisa el desarrollo y las características del CFI de Singapur, mientras en la sexta sección se desarrolla un sucinto análisis comparativo de la problemática que enfrentan ambas plazas después de la crisis asiática, y se intenta trazar algunas perspectivas.

\section{Tipos de CFIs y condiciones requeridas para su desarrollo}

Sin pretender ser exhaustiva, esta sección revisa las aportaciones de trabajos seleccionados que analizan la tipología de los CFIs, su papel, así como las condiciones necesarias para su desarrollo y el sostenimiento de su competitividad internacional.

Algunos trabajos han procurado definir criterios que permitan clasificar los centros financieros y determinar cuáles califican para esa posición. Un primer criterio pertinente puede ser constituido por la extensión del área geográfica que el centro sirve. Así, siguiendo a Reed, (1981, citado en Goldberg, 1996), los centros financieros pueden clasificarse en una jerarquía de cinco niveles, que constituyen también cinco etapas de desarrollo. Los tres primeros niveles implican una creciente importancia en el seno de un Estado-nación: desde el servicio proporcionado a una provincia, hasta el predominio a nivel nacional, pasando por un nivel intermedio de servicio a una región subnacional. Los dos niveles superiores implican la categoría de CFI, con servicios ofrecidos respectivamente a nivel regional supranacional (el país de origen y las naciones colindantes), y a nivel mundial para las plazas que se hallan en la cumbre de esta jerarquía.

Por otro lado, se puede utilizar el criterio de la nacionalidad de las contrapartes para diferenciar las operaciones de los centros financieros nacionales e internacionales. Aplicando este criterio, Montes (1999) distingue cuatro tipos de mercados financieros: el 1 intermedia fondos entre oferentes $y$ demandantes locales de capitales; el 2 efectúa esta operación entre oferentes extranjeros de capitales y demandantes locales; el 3 entre oferentes locales de capitales y demandantes extranjeros, y finalmente el 4 hace lo propio entre oferentes y demandantes extranjeros. Se desprende que para calificar como CFI, un mercado financiero debe mostrar una actividad 
sobresaliente en los tipos 2 a 4 de intermediación. Asimismo, el o los tipos de intermediación predominantes determinarán diferentes tipos de CFIs: orientados hacia adentro (tipo 2 predomina, caso de Manila o Bangkok), orientados hacia la colocación internacional de excesos de ahorro interno (tipo 3 predomina, caso de Tokio), o hacia los servicios de entrepôt financiero (tipo 4 predomina, casos de Hong Kong y Singapur).

Por último, se pueden diferenciar los CFIs en función de las actividades específicas que desempeñan: Reed (1981, citado por Goldberg, 1996) distingue los centros bancarios internacionales (CBIs) de los CFIs propiamente dichos, donde predominan las actividades centradas alrededor de los mercados financieros. Del análisis histórico que este autor llevó a cabo $^{3}$, se deduce que cada tipo de centro financiero presenta características peculiares, y que las tareas de intermediación bancaria que desempeñan los primeros, con frecuencia no facilitan su conversión en un CFI con actividades más diversificadas. En este esquema, la capacidad de atraer fondos y la preeminencia en actividades de gestión global de portafolio son las que otorgarían poder e influencia.

Ahora bien, ¿cuáles son las condiciones que determinarán que un centro financiero, dominante en una nación, adquiera un papel internacional? Sassen (1999, citado por Law, 1999) subraya la relevancia de la consolidación nacional de los mercados financieros, "que favorece las ciudades con mayores tenencias accionarias", y la ubicación, que sigue desempeñando un papel esencial, aun con los adelantos tecnológicos. El cuadro 1 proporciona los diversos criterios citados en tres trabajos recientes. De manera muy general se pueden identificar una serie de ventajas vinculadas con la ubicación -zona geográfica, huso horario, con la infraestructura-redes de transportes y comunicaciones avanzadas, sistemas legales y contables de calidad, infraestructura reglamentaria flexible e "ilustrada" ${ }^{4}$, y demás condiciones que permitan costos competitivosy con los recursos humanos: disponibilidad de personal calificado, fluidez en el idioma inglés.
Más allá de estos criterios de base, las instituciones financieras buscarán ubicarse en la proximidad de "yacimientos de ahorro", es decir de economías ricas en recursos para invertir, y cerca también de los usuarios de estos fondos: las grandes empresas, y en particular las empresas multinacionales (The Economist, 1999). Asimismo, las operaciones de comercio exterior propiciaran que los bancos "sigan" sus clientes en sus mercados más importantes; de allí la correlación positiva que se observa entre el nivel de importaciones de un país y su dinamismo en la actividad financiera (Goldberg, 1996).

Por otro lado, la concentración de instituciones financieras en un solo lugar tiende a generar "economías de aglomeración”; en otras palabras, "externalidades" positivas para los participantes que reducen sus costos y mejoran su competitividad, y que se convertirán en una ventaja adicional de los CFIs exitosos. Goldberg (1996) las clasifica en dos niveles: economías de escala, internas a la industria, que repercuten favorablemente sobre los costos de las instituciones financieras individuales, y economías externas a la industria, relacionadas con la infraestructura de servicios y humana. En consecuencia "La concentración de los principales participantes del mercado en los centros financieros líderes ha reforzado la tendencia a la concentración de la innovación financiera en estos lugares, dando a las firmas allí presentes una ventaja competitiva significativa. Estar en centros innovadores resulta crucial para un aprendizaje rápido de los nuevos instrumentos financieros. Asimismo, sin el personal financiero calificado y los servicios de apoyo legal y de negocios disponibles en estos lugares, las empresas no pueden proporcionar a sus clientes el amplio rango de servicios complejos que éstos necesitan” (Goldberg, 1996: 388-389).

En suma, la revisión de los factores que favorecen el surgimiento y el desarrollo de un CFI muestra el carácter altamente competitivo de esta posición, y la combinación de "fuerzas del mercado" y de reglamentaciones y atractivos establecidos por políticas públicas, que 


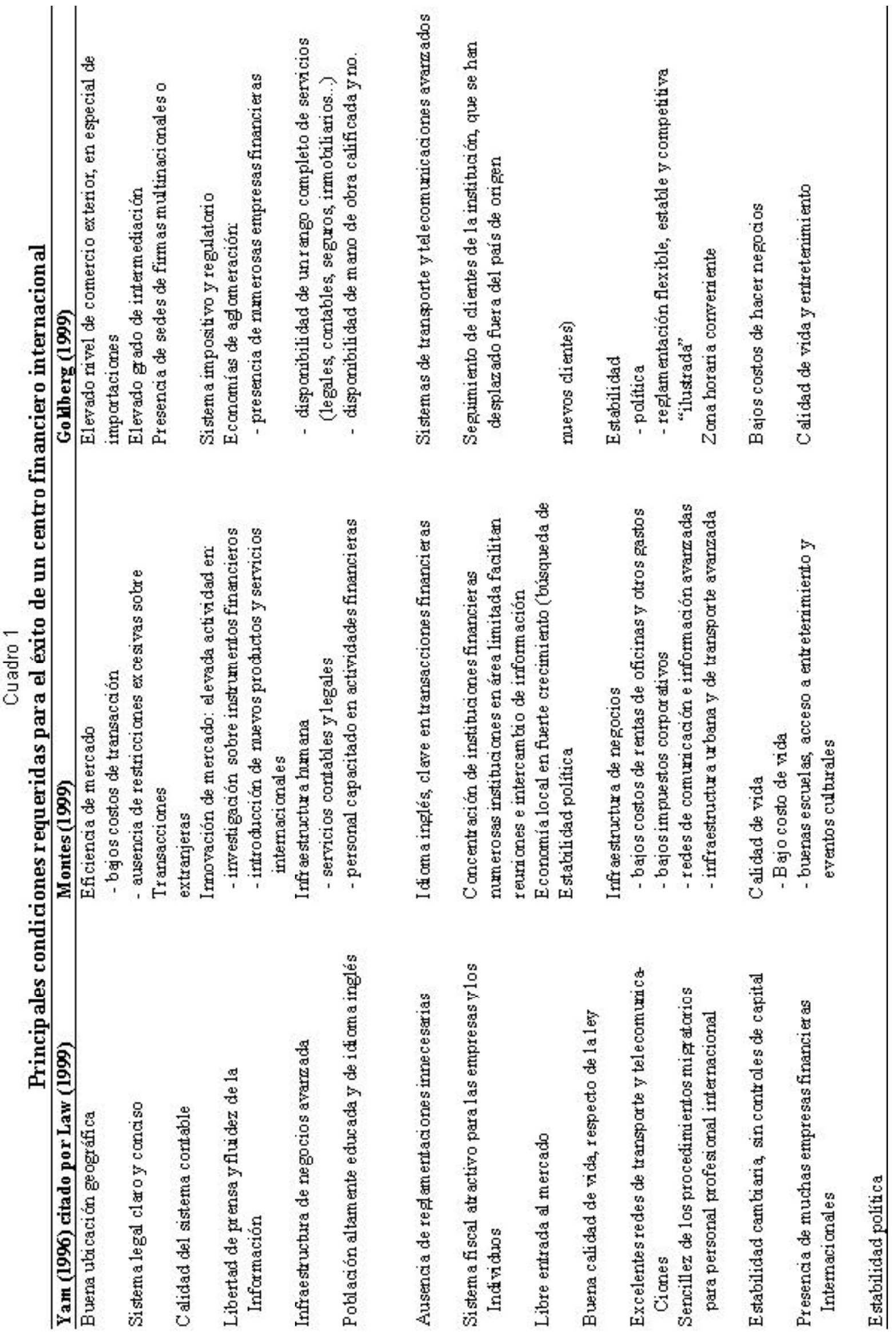


contribuye a establecerlo y a reforzar su competitividad. En la sección siguiente, se analizarán los factores que propiciaron el surgimiento de Hong Kong como CFI, su especialización y ventajas específicas.

\section{El centro financiero internacional de Hong Kong: pautas de desarrollo}

El CFI de Hong Kong ocupa un lugar preeminente en Asia-Pacífico y figura entre los primeros a nivel mundial. Presenta en mayor o menor grado las características señaladas en el cuadro 1, pero siguiendo a Crawford (2001: 49), las claves de su éxito residirían: “...en primer lugar, [en] la capacidad de Hong Kong de proveer un ámbito financiero competitivo - "occidental" de hecho-favorable a los intereses y las demandas de las firmas financieras globales ... En segundo lugar, y de la misma importancia, es el estatus de Hong Kong en tanto puente hacia China y como centro del capital étnicamente chino". En consecuencia, se desprende que el porvenir de Hong Kong depende estrechamente de la calidad de su relación con China y, a su vez, del futuro de las relaciones económicas y políticas de este país con el resto del mundo ${ }^{5}$.

El cuadro 2 concentra los rangos alcanzados por Hong Kong en sus diversas actividades financieras, es decir la banca, los mercados de divisas, de oro y de derivados financieros, el mercado accionario, los seguros y la gestión de activos. Significativamente, el mercado de bonos, insuficientemente desarrollado, no figura en el cuadro. En pocas palabras, el cuadro indica que la preeminencia del territorio es especialmente clara en las actividades bancarias y en el mercado del oro (primera o segunda posición en Asia, entre la segunda y la sexta a nivel mundial), y destacada también en el mercado de divisas y en los derivados (tercer o cuarto rango en Asia, entre la quinta y la octava posición a nivel mundial).

En lo que sigue, presentaremos la estrategia seguida por el territorio para desarrollar su esfera financiera y analizaremos las características actuales de este sector.

\section{La estrategia de desarrollo financiero temprano}

Con excepción del periodo anterior al año 1964, la estrategia aplicada por las autoridades del territorio, en materia financiera, se alejó tanto del liberalismo pleno como de la "represión financiera" (Cheng y Ng, 1999). Si bien las políticas fueron modificándose a lo largo de los años, conservaron algunos elementos centrales constantes, como la plena libertad cambiaria y de flujos de capitales, la ausencia de barreras entre las operaciones orientadas hacia el mercado interno y la intermediación internacional; asimismo, ciertos instrumentos típicos de la represión financiera, como el crédito selectivo o la intervención pública en la asignación de recursos, no se utilizaron jamás. Sin embargo, el esfuerzo inicial por atraer bancos extranjeros en condiciones de amplia competencia se matizó luego con una mayor preocupación por la estabilidad del sistema, la que llevó a las autoridades a reforzar paulatinamente la reglamentación y la supervisión, y a tolerar arreglos que limitaron la competencia, en particular a través de las tasas de interés.

Tres etapas pueden diferenciarse en las políticas aplicadas al sector financiero de Hong Kong (Cheng y Ng, 1999): 1) antes de 1964 predominaron políticas de laissez-faire;2) entre 1964 y 1981 se dio una etapa de "restricción financiera" y, posteriormente 3) se lanzó un amplio movimiento de reformas fundamentales que dieron sus características centrales a la esfera financiera actual.

El periodo anterior a 1964 puede considerarse extremadamente liberal, con pocos requisitos adicionales a la compra de una licencia y el pago de un impuesto anual para establecer un banco. Esto favoreció un crecimiento muy rápido del sector, interrumpido en 1964 por un episodio de falta de liquidez en algunos bancos, vinculado con una concentración excesiva de sus inversiones en el sector inmobiliario, y, en 1967, por una crisis bancaria, que generó un primer movimiento de consolidación del sector. Estos eventos 
Clasificación del CFI de Hong Kong (1995)

Categorías

Rango en Asia Rango mundial

\section{Banca}

Número de bancos extranjeros

$\begin{array}{ll}1 & 2 \\ 2 & 4 \\ 2 & 5 \\ 2 & 6 \\ 2 & 4 \\ 1 & 2 \\ 1 & 4\end{array}$

Activos externos de los bancos

2

Pasivos externos de los bancos

Activos interbancarios internacionales

Pasivos interbancarios internacionales

Crédito internacional a entidades no-bancarias

Préstamos sindicados y emisión de notas (1994)

\section{Mercado de divisas}

Actividad diaria neta

\section{Mercado de derivados}

Actividad diaria neta en contratos de divisas

Actividad diaria neta en contratos de tasas de interés

Total

$\begin{array}{ll}3 & 5 \\ 4 & 8 \\ 3 & 7\end{array}$

\section{Mercado accionario}

Capitalización de mercado

Monto intercambiado

29

Número de empresas domésticas que cotizan

$4 \quad 11$

$7 \quad 16$

Mercado de oro

1

4

\section{Seguros}

Número de compañías de seguros

Ingreso por concepto de primas

Número de actuarios calificados

Gestión de fondos

2

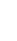

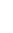

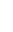

Fuente: Jao (1997) citado en Law (1999)

propiciaron una primera reforma bancaria y un reforzamiento inicial de la reglamentación y de la supervisión (ibid.). Establecieron también un patrón, en el cual la especulación inmobiliaria, y luego bursátil, crea problemas bancarios que suscitan a su vez un fortalecimiento ad hoc de la infraestructura y de las prácticas de supervisión.
En consecuencia, el período que abarca los años que van desde 1964 hasta 1981 se inicia con una reforma bancaria, que incluye la creación de una comisión de supervisión bancaria (Office of the Commissioner of Banking), el fortalecimiento de los requisitos de capitalización y de liquidez bancaria, y la prohibición de las inversiones de alto riesgo. Comprende también dos medidas destinadas a 
reducir el grado de competencia en el sistema bancario, estableciéndose un régimen de "restricción financiera". En primer lugar, se introduce una moratoria de licencias bancarias, reemplazada en 1978 por licencias que limitan los nuevos participantes extranjeros a abrir una sola sucursal. En segundo lugar, la introducción del Acuerdo sobre Tasas de Interés de parte de la asociación de bancos (Exchange Bank Association), tolerada por las autoridades, estableció topes a las tasas de depósito, eliminando toda competencia a través de los precios (ibid.).

Ahora bien, la introducción de una reglamentación más severa para las operaciones bancarias suscitó el crecimiento de un mercado "informal", a través de la creación de instituciones más "libres": las compañías de depósito ${ }^{7}$ La proliferación de estos establecimientos -eran alrededor de 2000 en 1973- llevó a las autoridades a crear en 1976 una reglamentación específica que redujo significativamente su número, pues sólo 200 pudieron registrarse formalmente (ibid.).

\section{Las políticas de desarrollo financiero desde 1981}

A partir de 1981, las políticas aplicadas a la esfera financiera establecen los elementos centrales del sistema financiero actual: la liberalización gradual y el fortalecimiento de la regulación y de la supervisión de un sistema bancario de tres niveles, la creación de la Autoridad Monetaria de Hong Kong y el fomento a los mercados financieros.

Entre estas medidas figura, de manera prominente, el establecimiento del arreglo cambiario vigente hasta la fecha: el sistema de tipo de cambio atado al dólar de Estados Unidos (US\$). En efecto, Hong Kong había abandonado un sistema previo de caja de convertibilidad, para adoptar, en 1974, un tipo de cambio flexible, siguiendo la introducción del régimen de flotación para las grandes monedas, consecutivo al desplome del sistema de cambios fijos de Bretton Woods. En medio de la crisis de 19821983, el territorio regresó a un sistema de caja de convertibilidad, con un régimen de cambio dual (linked exchange rate regime) en el cual el dólar de Hong Kong (HK\$) tiene una paridad fija con el dólar de Estados Unidos. Se considera que en la economía hongkonesa, este régimen de cambio puede contribuir a reducir la volatilidad excesiva del tipo de cambio y por tanto la incertidumbre, facilitando de esta manera la inversión (Cheng y Ng, 1999). Esta paridad se ha mantenido hasta la fecha, superando diversos ataques especulativos gracias a las medidas muy enérgicas que fueron implementadas para contrarrestarlos durante la crisis asiática ${ }^{8}$.

En el ámbito bancario, desde 1981 la moratoria de licencias bancarias ha sido levantada, y el sistema ha sido reorganizado en tres niveles: los bancos con licencia, los bancos con licencia restringida y las compañías de depósito registradas; esta reforma procuró incorporar las entidades informales al sistema bancario formal, creando los dos últimos tipos de instituciones, que ven limitada su capacidad de recibir depósitos del público. La reglamentación y la supervisión han sido notablemente reforzadas en respuesta a una serie de colapsos bancarios que se produjeron en $1982-1983^{9}$ y en $1985-1986$. Entre las medidas introducidas cabe resaltar el fortalecimiento de la comisión de supervisión bancaria en 1986 y la introducción, en 1989, de los requisitos de capitalización bancaria de Basilea -capitalización mínima de 8\% de los activos ponderados por niveles de riesgo-( $i b i d$.).

Nuevas reformas fueron emprendidas, después de la crisis asiática, en respuesta a las recomendaciones formuladas por un estudio ("Banking Sector Consultancy Study") encargado por la Autoridad Monetaria a las consultoras KPMG y Barents justo antes del estallido de la crisis. Anticipando un contexto, que se vive desde la crisis, de menor crecimiento de los préstamos y de márgenes de ganancia más estrechos, el reporte condujo a las autoridades a lanzar un paquete de reformas que tenía dos objetivos centrales: "ayudar a mejorar la eficiencia y la innovación en el sistema bancario a través de una mayor 
competencia..." y "elevar la capacidad de atracción de Hong Kong en tanto centro financiero internacional" (Carse, 2001: 2). Estas medidas,destinadas a favorecer la competencia,comprenden la eliminación de restricciones sobre el número de oficinas que pueden abrir los bancos extranjeros y la invitación a los bancos de licencia restringida para que participen en el sistema interbancario de pagos en tiempo real.

Finalmente, todas las medidas incluyeron la aceleración del proceso de desreglamentación de las tasas de interés. La paulatina supresión de los topes de tasas de interés implementados por la Asociación Bancaria de Hong Kong ${ }^{10}$ se había iniciado tardíamente y con mucha prudencia en 1994. Las reformas completaron este proceso, que culminó en julio de 2001, con la eliminación de los topes sobre tasas de interés de depósitos de ahorro y de la prohibición de remunerar las cuentas corrientes (ibid.: 3). Las autoridades esperan que la desreglamentación podría llevar a los bancos locales hacia un proceso de concentración.

Las dos ultimas décadas del siglo XX fueron también importantes para la reglamentación y supervisión del mercado accionario: siguiendo un primer intento de fortalecimiento de la reglamentación implementado después de un auge en el periodo 1969-1973, durante el cual se establecieron la Comisión de Valores y la Oficina del Comisionado a los Valores (respectivamente Securities Commission y Office of the Commissioner for Securities), el año 1986 señaló la consolidación de las cuatro bolsas existentes en una sola Bolsa de Valores de Hong Kong (HKSE). El posterior desplome de la bolsa en 1987 justificó una reforma regulatoria y la creación de una Comisión de Valores y Futuros autónoma. En la década de 1990, se amplió el alcance de los mercados al introducir nuevos instrumentos como las opciones sobre acciones, negociadas de manera electrónica. Asimismo, se buscó que estos instrumentos tuvieran un reconocimiento internacional.
Una iniciativa de gran importancia consistió en abrir a empresas chinas calificadas la posibilidad de financiarse en Hong Kong, creándose la sección de acciones "H" oredchips. Éstas son compañías que tienen su sede en Hong Kong, que cotizan y solicitan fondos en el HKSE, pero que pertenecen a empresas matrices y a autoridades de China continental (Crawford, 2001:57). A mediados de los años 90, numerosas empresas chinas empezaron a cotizar en el HKSE de esta manera y, en 1996 y 1997, antes de la devolución de Hong Kong a China, el mercado de red chips experimentó un alza especulativa extraordinaria, que multiplicó hasta en 20 o 30 veces el valor de mercado de estas compañías (ibid: 58). Esta iniciativa fortaleció el papel de intermediario del territorio "al poner los mercados emergentes de China en contacto directo con el sistema financiero global" (ibid.: 57).

Finalmente, en los primeros meses de 2000, el HKSE se fusionó con la Bolsa de Futuros de Hong Kong y con tres casas de liquidación (clearing houses) para formar una nueva entidad, nombrada Hong Kong Stock Exchanges and Clearing (HKEx), que adoptó la forma legal de sociedad anónima ${ }^{11}$. Esta fusión tuvo como principal objetivo proporcionar a los emisores e intermediarios un mercado de mayor tamaño, y acercar a Hong Kong al modelo seguido en América del Norte y Europa (Crawford, 2001: $52)$.

De importancia central, para la infraestructura reguladora y supervisora, fue la creación, en 1993, de la Autoridad Monetaria de Hong Kong (AMHK), un banco central de facto sobre la base de la fusión del Fondo de Cambios y de la Comisión de Supervisión Bancaria. Esta nueva institución no solamente ha contribuido a reforzar el marco regulatorio sino que ha permitido fomentar el desarrollo del mercado de títulos de deuda. En efecto, la AMHK ha implementado un programa de emisión de papeles del Fondo de Cambios, creándose una serie de instrumentos de deuda pública que, al proporcionar tasas de interés de referencia, (benchmark), han facilitado el desarrollo de mercados de títulos privados ${ }^{12}$. 
Entre otras medidas de fomento de estos mercados, cabe resaltar la introducción de un sistema de liquidación eficiente, en enero de $1994^{13}$,y la creación de la Corporación Hipotecaria en 1997, que facilitó la ‘bursatilización' de los préstamos hipotecarios (Cheng \& Ng, 1999). En los últimos tiempos, los esfuerzos de las autoridades se han concentrado en mejorar la transparencia de la información proveída por las instituciones financieras y en reforzar la seguridad de la plaza.

\section{Bancos y mercados financieros en Hong Kong: de 1980 a 2001}

En razón de su posición en tanto CFI, Hong Kong posee un grado de monetización muy elevado (gráfico 1): la razón M2/PIB superó $200 \%$ del PIB desde inicios de los años 90 se ha elevado aún más después de la crisis asiática (268\% del PIB en 1999). El crecimiento del crédito total y del crédito al sector privado ha sido también fuerte hasta 1997, cuando estas dos razones culminaron respectivamente en 168 y $175 \%$ del PIB de la entidad. Después de esta fecha, las razones de monetización y de crédito divergen, contrayéndose las últimas, e indicando de esta manera los problemas que atraviesa el sector bancario, y que analizaremos más adelante.

La expansión histórica y la problemática actual del sector bancario se reflejan también en su composición. En efecto, si exceptuamos

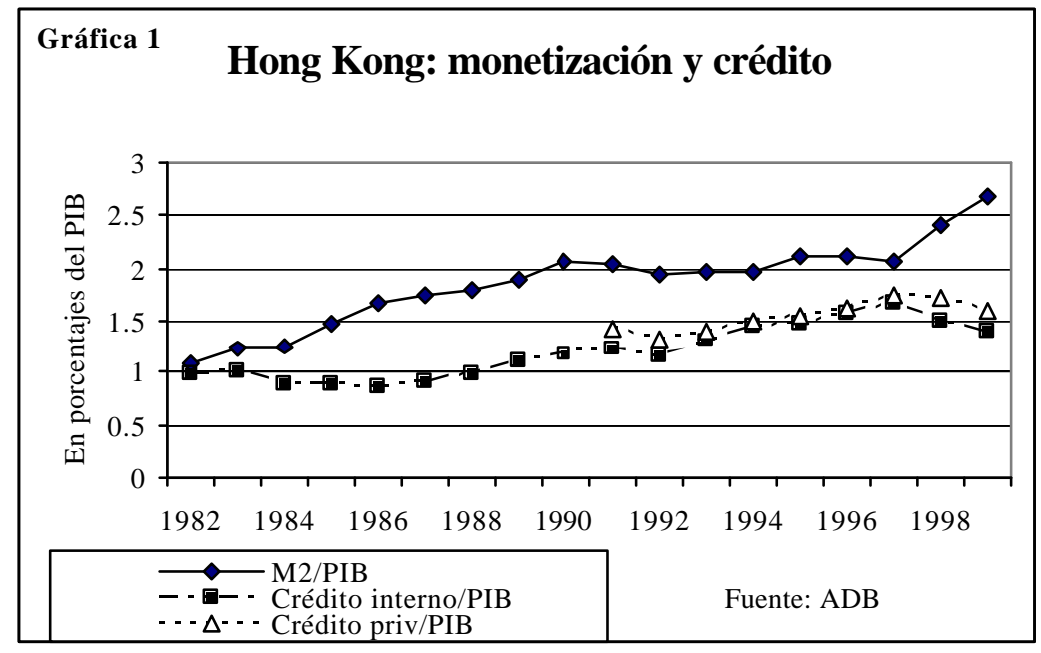

las compañías de depósito ${ }^{14}$, la composición del sistema bancario de tres niveles muestra un proceso continuo de incorporación de nuevas instituciones desde 1969 hasta 1997, y una contracción rápida del número de entidades desde la crisis asiática hasta junio de 2002 (cuadro 3). El número total de instituciones del sistema, que alcanzó el nivel de 361 en 1997, había caído a 229 en junio de 2002 , disminución que se ha dado en todos los segmentos. Las entidades más importantes del sistema, los bancos con licencia, pasaron de 180, entre 1995 y 1997, a 133 en junio de 2002 , y esta reducción de dio principalmente en la banca extranjera (de 149 a 106): Crawford (2001: 49) señala que en 1995, 85 de los 100 primeros bancos mundiales estaban presentes en Hong Kong, número que había caído a 79 en $1998^{15}$. El primer banco de la economía es de hecho uno multinacional con gran presencia en toda la región: el Hong Kong and Shanghai Banking Corporation (HSBC). Reducciones importantes se observan también en las oficinas de representación de bancos extranjeros (de 159 a 102) y en menor medida en el número de bancos con licencia restringida (de 63 a 48).

La contracción en el número de instituciones financieras presentes en Hong Kong se puede atribuir, en primer lugar, a las consecuencias de la crisis asiática, pero también a la ola de fusiones y adquisiciones en la esfera bancaria internacional y en el ámbito financiero local. Así, en octubre de 2001, la fusión del Banco de China (Hong Kong), entidad perteneciente al homónimo Banco de China, uno de los cuatros grandes bancos de la República Popular China (RPCh), con 7 sucursales locales de bancos chinos $\mathrm{y}$ dos instituciones de bancos locales, creó la segunda entidad bancaria en términos de activos, relegando en tercera posición al Banco Hang Seng (South China Morning Post (SCMP), 2,10,2001).

Ahora bien, la importancia relativa de cada tipo de institución, en el sistema bancario de tres 
Cuadro 3

\begin{tabular}{|c|c|c|c|c|c|c|c|c|}
\hline & \multicolumn{2}{|c|}{ Bancos con Licencia } & \multicolumn{2}{|c|}{$\begin{array}{l}\text { Bancos con licencia } \\
\text { restringida }\end{array}$} & \multicolumn{2}{|c|}{ Compañías de depósito } & \multirow{2}{*}{$\begin{array}{c}\text { Instituciones } \\
\text { autorizadas } \\
\text { TOTAL }\end{array}$} & \multirow{2}{*}{$\begin{array}{l}\text { Oficinas de } \\
\text { representación } \\
\text { local }\end{array}$} \\
\hline & $\begin{array}{l}\text { Con sede en } \\
\text { Hong Kong }\end{array}$ & $\begin{array}{l}\text { Con sede en } \\
\text { el extranjero }\end{array}$ & $\begin{array}{l}\text { Con sede en } \\
\text { Hong Kong }\end{array}$ & $\begin{array}{l}\text { Con sede en } \\
\text { el extranjero }\end{array}$ & $\begin{array}{l}\text { Con sede en } \\
\text { Hong Kong }\end{array}$ & $\begin{array}{l}\text { Con sede en } \\
\text { el extranjero }\end{array}$ & & \\
\hline \multicolumn{9}{|l|}{ A fines de } \\
\hline 1969 & \multicolumn{2}{|c|}{73} & \multicolumn{2}{|c|}{ nd } & \multicolumn{2}{|c|}{ nd } & nd & 21 \\
\hline 1978 & \multicolumn{2}{|c|}{$\begin{array}{c}88 \\
121\end{array}$} & \multicolumn{2}{|c|}{ nd } & \multicolumn{2}{|c|}{241} & nd & 100 \\
\hline 1981 & \multirow{2}{*}{\multicolumn{2}{|c|}{$\begin{array}{l}121 \\
143\end{array}$}} & \multicolumn{2}{|c|}{ nd } & \multicolumn{2}{|c|}{350} & 471 & nd \\
\hline 1985 & & & \multirow{2}{*}{\multicolumn{2}{|c|}{$\begin{array}{l}35 \\
44\end{array}$}} & \multicolumn{2}{|c|}{278} & 456 & nd \\
\hline 1990 & \multicolumn{2}{|c|}{$\begin{array}{l}143 \\
166\end{array}$} & & & \multicolumn{2}{|c|}{190} & 400 & nd \\
\hline 1995 & \multicolumn{2}{|c|}{166} & \multicolumn{2}{|c|}{44} & 134 & 3 & 380 & 157 \\
\hline 1997 & 31 & 149 & 39 & 27 & 113 & 2 & 361 & 159 \\
\hline 1998 & 31 & 141 & 35 & 25 & 99 & 2 & 333 & 141 \\
\hline 1999 & 31 & 125 & 33 & 25 & 71 & 0 & 285 & 128 \\
\hline 2000 & 31 & 123 & 28 & 20 & 61 & 0 & 263 & 118 \\
\hline 2001 & 29 & 118 & 29 & 20 & 54 & 0 & 250 & 111 \\
\hline 2002 (junio) & 27 & 106 & 29 & 19 & 48 & 0 & 229 & 102 \\
\hline
\end{tabular}

niveles, es muy desigual. Un primer indicador lo constituye la participación porcentual de los diferentes tipos de entidades en la captación de depósitos bancarios (gráfica 2). Desde 1980, el peso relativo de los bancos ha ido fortaleciéndose conforme se expandía la captación (medida en dólares de Hong Kong corrientes). Como resultado de esta tendencia, las compañías de depósitos, que captaban alrededor de la tercera parte de los depósitos en 1980-81, pasaron a captar menos de 5\% del total desde 1987, y menos de $1 \%$ desde 1993-94, mientras los bancos con licencia restringida, que captaban alrededor de 5\% de los depósitos en 1983-84 tienen en la actualidad (2001-2002) menos de 1\% del total. En otras palabras, los diferentes paquetes de reformas legales aplicados desde los años 80 han favorecido la expansión de las instituciones

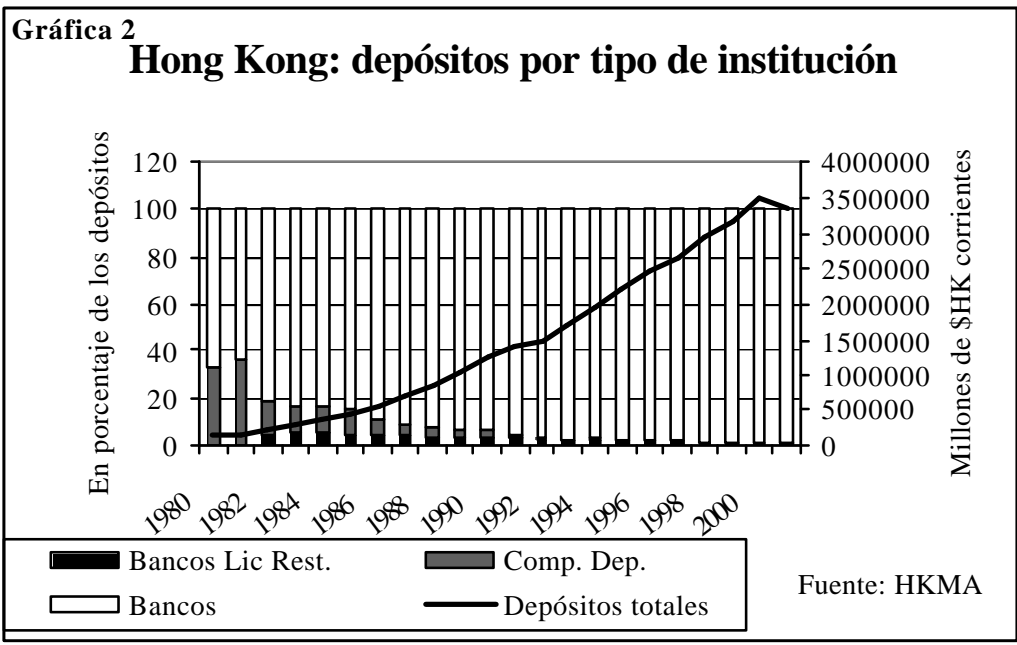

dotadas de mayores prerrogativas, que son también las más internacionalizadas.

Paralelamente a esta evolución tuvo lugar un proceso de diversificación de las divisas en las cuales los depósitos y préstamos están denominados, lo cual refleja también el crecimiento del papel internacional del sistema bancario de Hong Kong. De manera característica, este proceso ha sido revertido en parte en los años 90, sin que la crisis asiática constituya el factor de mayor peso. La gráfica 3 muestra que en 1980 los depósitos estaban denominados en $\mathrm{HK} \$$ a altura de $86 \%$, contra solo $12 \%$ para el US\$ y $2 \%$ para las demás divisas (yen japonés, monedas europeas). Entre esta fecha y 1991 se desarrolla un rápido proceso de internacionalización de los depósitos: en mayo de este año, los depósitos en HK\$ solo representaban $42 \%$ del total, contra $24 \%$ para el US $\$$ y $34 \%$ para las demás monedas. Desde mediados de 1991, la tendencia en curso desde 1980 se revierte debido a la disminuida participación de las divisas extranjeras, no US\$. A partir de finales de 1997, la crisis asiática acelera más esta evolución, a la vez que eleva la participación relativa del US\$ en los depósitos (gráfica 4). 


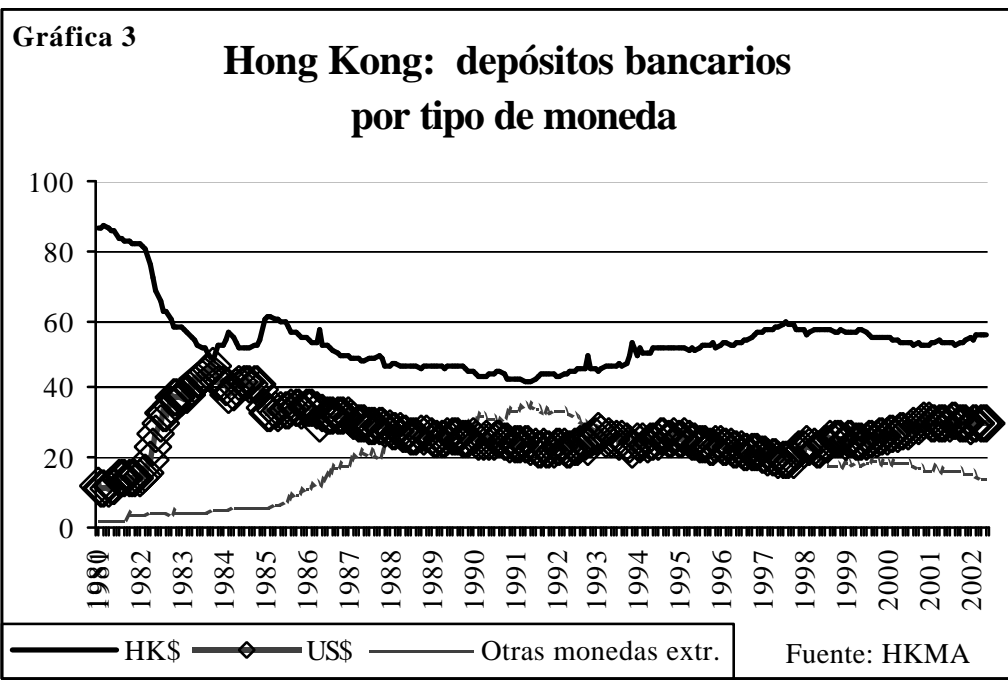

tendencia en los años 90 puede deberse, en parte, a fenómenos cambiarios, específicamente al proceso de apreciación del US\$ y de las divisas asiáticas definidas en relación con esta divisa -en particular el propio $\mathrm{HK} \$-$, frente al yen y a las divisas europeas. En segundo lugar, dichos cambios están vinculados con una modificación, más bien un deterioro del entorno regional, que afectó al papel desempeñado por Hong Kong. Esta afirmación se sustenta en el cuadro 4, que muestra los cambios en la posición internacional (activos y pasivos) del CFI frente a sus

Una evolución de tendencia similar, aunque más pronunciada, se observa en los préstamos hechos por bancos del centro financiero: la proporción de préstamos denominados en US $\$$ se eleva de $43.5 \%$ del total en 1980 a $67.7 \%$ en 1991, para luego contraerse a $24.6 \%$ a fines de 2001. A partir de 1995, esta modificación en la composición de los préstamos por monedas está asociada a una disminución, en términos absolutos, de los préstamos denominados en divisas, que se acelera después de la crisis: en 2001, éstos solo representaban alrededor de la quinta parte del valor alcanzado en 1995 (gráfica 5). Desde 1998 se produce igualmente una disminución de los préstamos hechos en moneda local. La gráfica 6 confirma que esta evolución refleja una drástica caída de los préstamos internacionales del centro financiero, misma que empezó antes de la crisis asiática, entre 1995 y 1996, así como una disminución proporcionalmente menor de los créditos concedidos a agentes residentes, producto de la crisis experimentada por la economía de Hong Kong a fines de 1997.

Está evidentemente más allá del objetivo de este artículo explicar en profundidad esta evolución; nos limitaremos a sustentar un par de hipótesis que nos parecen centrales. En primer lugar, el cambio de principales contrapartes: la RPCh, Japón, Singapur, Corea el Sur, los Estados Unidos y el Reino Unido, que representan conjuntamente una proporción elevada de los activos y pasivos externos de Hong Kong: alrededor de $80 \%$ en 1997, y una proporción cercana al 60\% en 2002.

\section{Cuadro 4}

Ahora bien, la fuerte disminución de activos y pasivos que documenta el cuadro tiene su principal origen en la economía japonesa: en particular, los pasivos de entidades de Hong Kong con bancos japoneses caen en $82 \%$ en el período, mientras que los activos de entidades registradas en el CFI con clientes no-bancarios japoneses lo hacen en $88 \%{ }^{16}$. El Banco de Pagos Internacionales ya registraba este cambio en
Gráfica 4

Hong Kong: depósitos bancarios, dic. 2001 (por tipo de moneda)

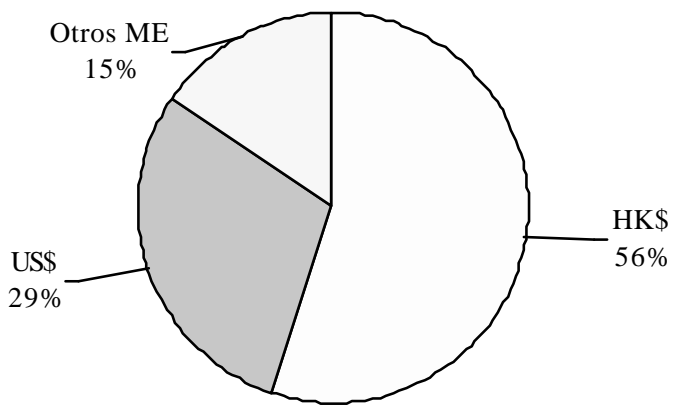

Fuente: HKMA 
Cuadro 4

Hong Kong: Evolución de los activos y pasivos externos, países seleccionados (dic. 1997-junio 2002)

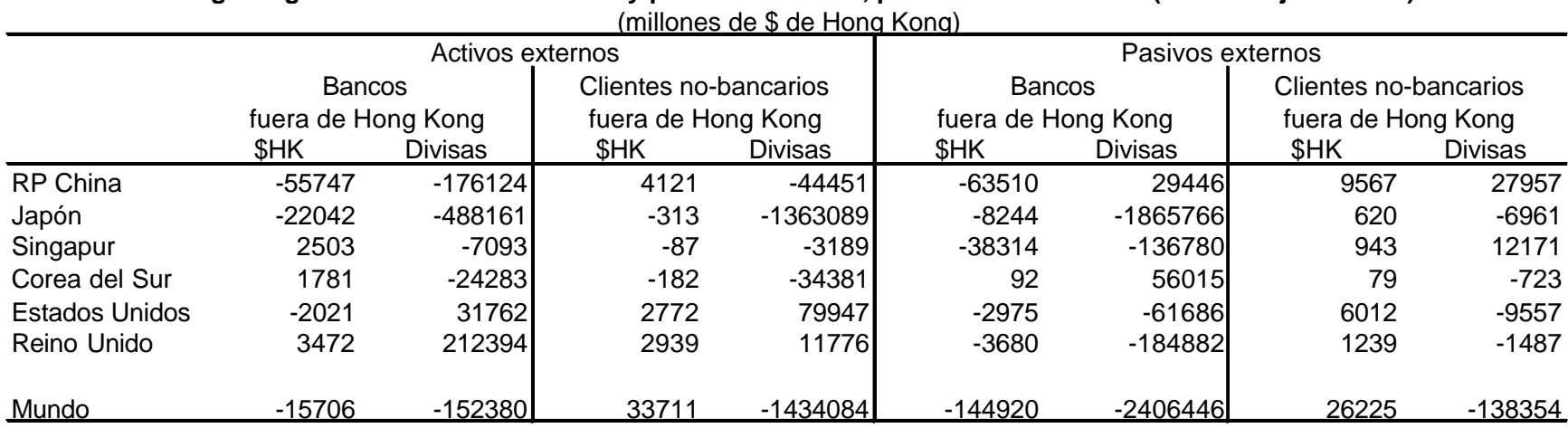

Fuente: HKMA

1999 (McCaulay \& Mo, 1999: 13) y lo atribuía a la crisis bancaria japonesa. El sistema bancario de Hong Kong servía como una "extensión" del sistema bancario japonés, mayormente con fines de evadir reglamentaciones vigentes en el archipiélago: "los fondos 'hacían el viaje redondo' de Japón a Hong Kong, bajo la forma de pasivos bancarios, y de Hong Kong a Japón, sobretodo como préstamos a clientes no-bancarios" (ibid.). Esto llevó a que $60 \%$ de los activos bancarios internacionales del CFI hayan estado en manos de sucursales de bancos japoneses. Después de 1997, tres factores se combinan para interrumpir este circuito: en primer lugar, la debilidad de la demanda de crédito en Japón; en segundo lugar, el alza del "premio Japón"17, que, al elevar el costo del

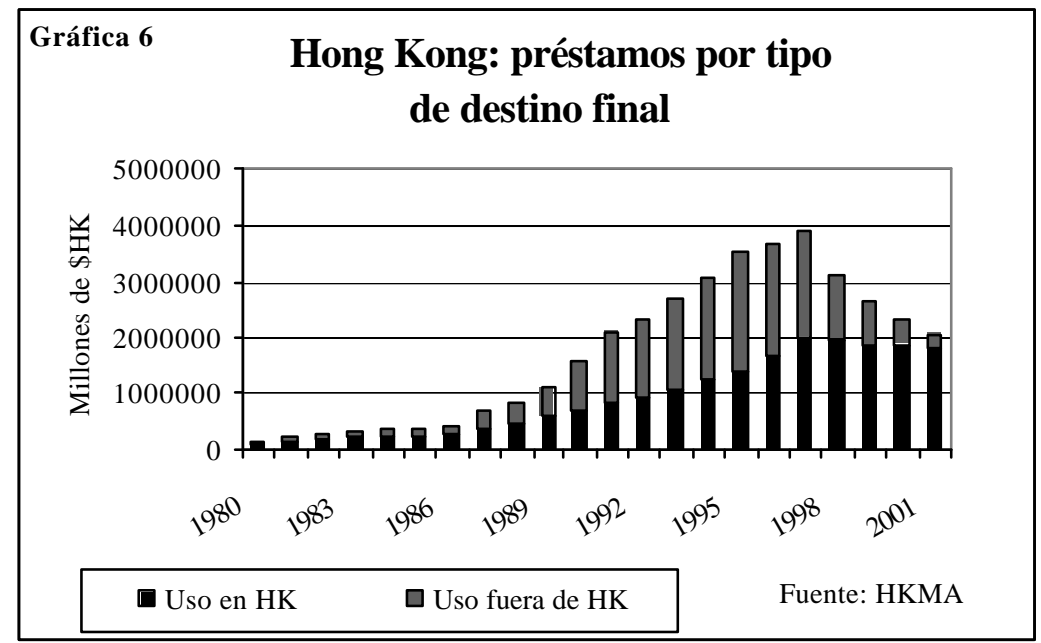

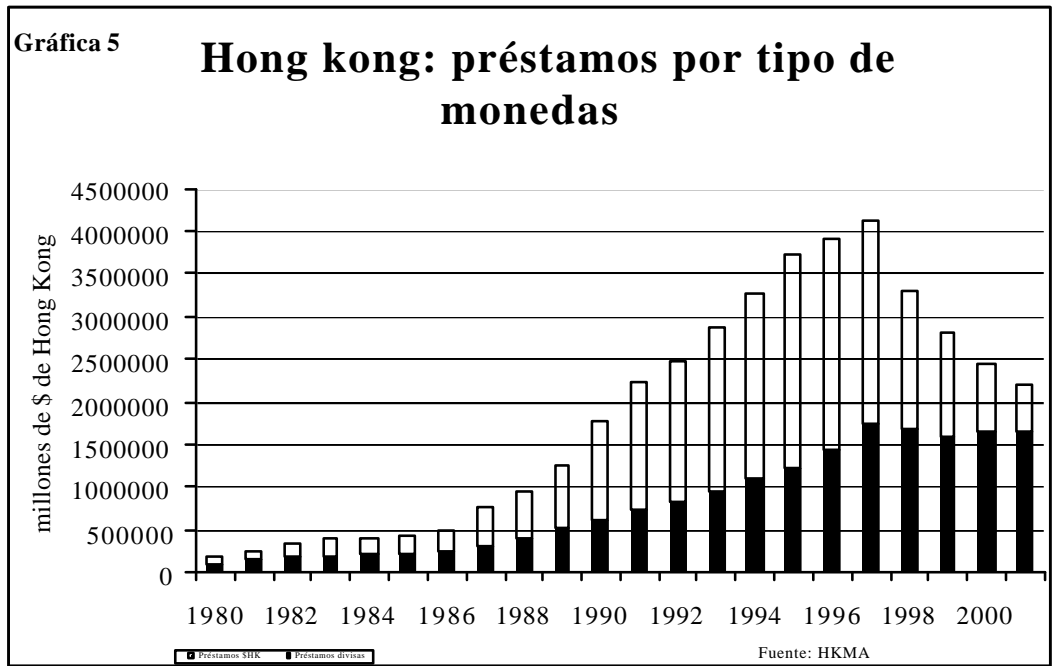

financiamiento en US\$, redujo la disposición de las entidades de este país a endeudarse en esta moneda, y, finalmente, el esfuerzo realizado por los bancos japoneses para mejorar sus razones de capitalización. Como resultado se estima que el CFI de Hong Kong ha sufrido proporcionalmente más que el de Singapur la crisis regional.

Cabe señalar también que la crisis afectó las relaciones financieras del CFI con la RPCh, traduciéndose, en particular, en una elevación de los pasivos netos del primero frente a la segunda. En efecto, se produjo, por un lado, una caída de los activos financieros de entidades de Hong Kong en China continental, atribuible a la disminución de préstamos de la banca internacional que siguió a la 
${ }^{\text {Gráfica }}{ }^{7}$ Hong Kong: compañías y capitalización del mercado accionario

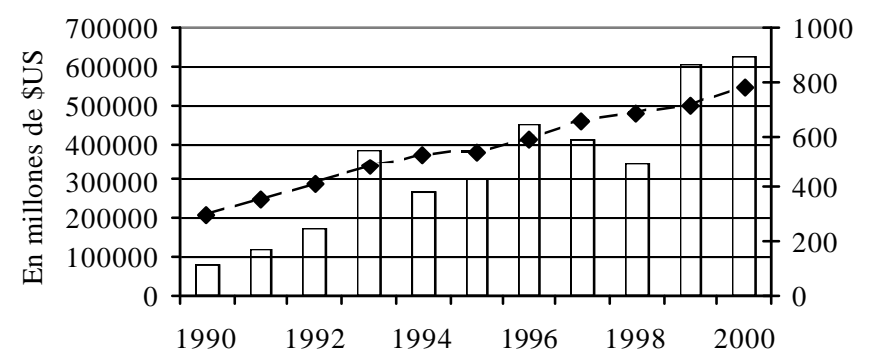

Fuente: FIBV a inicios de $1991^{19}$, obviamente un factor de riesgo para el sistema bancario local.

Por último, cabe señalar el desempeño de la bolsa de valores de Hong Kong. Esta ha ido creciendo en tamaño a lo largo del periodo considerado, en particular en los años 90. Las compañías registradas en Hong Kong, que cotizan en la bolsa, pasaron de 299 en 1990 a 779 en 2000 , mientras la capitalización de mercado se elevaba de 83385.9 a 623397.7 millones de US\$. Parte de este resultado se debe a una quiebra del Guandong International Trust and Investment Corporation (GITIC) en $1998^{18}$. Simultáneamente, ciertos cambios en las reglamentaciones chinas permitían un alza de los depósitos de empresas chinas en el CFI (Shi, 2002).

Para concluir con este punto, es necesario subrayar el impacto de la crisis en el ámbito bancario interno. El deterioro del contexto macroeconómico en 2001 -con un crecimiento cercano a cero, una nueva caída de las exportaciones y una deflación de los precios internos de más de tres años de duración- ha vuelto a actualizar la problemática de la cartera vencida bancaria. En efecto, ésta ha ido elevándose sin por ello poner en riesgo la solidez del sistema: representaba $6.8 \%$ de los créditos totales a fines de 2001. En particular, la deflación y la caída de los precios del sector inmobiliario han puesto en situación muy difícil a parte de los deudores hipotecarios, puesto que el valor de sus propiedades es ahora inferior al de sus deudas (una situación de negative equity o riqueza negativa). Esto reviste especial importancia si consideramos que los préstamos bancarios locales han ido concentrándose paulatinamente en el sector inmobiliario en los años 90; en junio de 2002, constituían 58\% de los créditos locales, contra $37 \%$

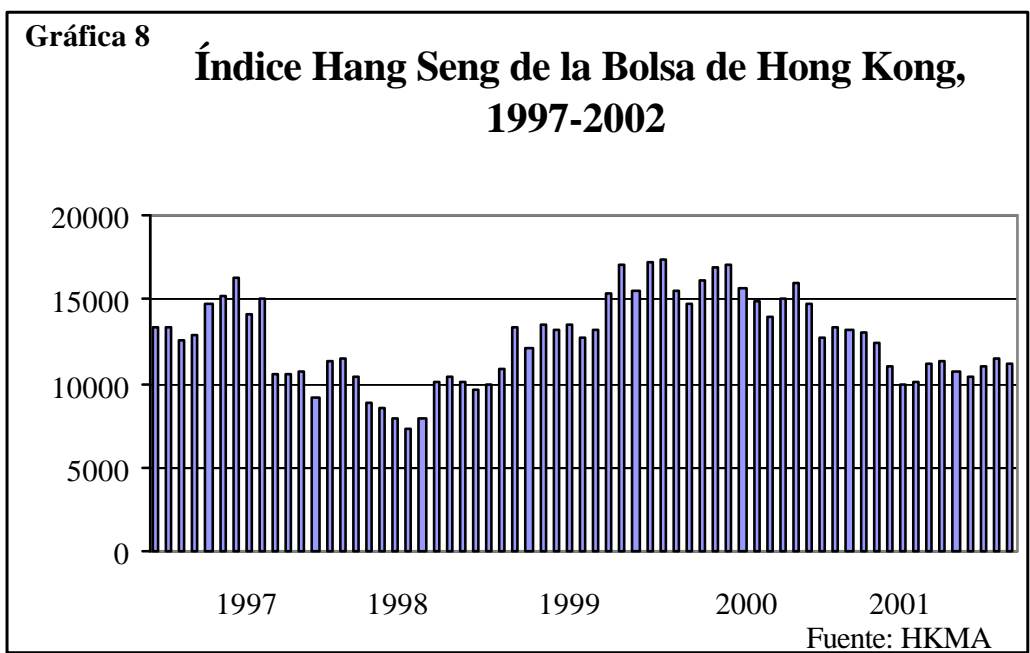




\section{Conclusión}

En suma, el rápido desarrollo de la plaza financiera de Hong Kong, en los últimos 30 años, está ahora cuestionado: a la crisis que golpea con fuerza desde 1997 al sector bancario internacional se han sumado los efectos de la crisis bursátil mundial, la mayor volatilidad financiera internacional y la deteriorada coyuntura económica global. Las amenazas al sector financiero han llevado a las autoridades a una actividad intensa en su búsqueda de caminos que permitan elevar la competitividad del CFI en la arena financiera global.

En primer lugar, las medidas y propuestas oficiales apuntan hacia una mayor desreglamentación: entre las últimas cabe mencionar la disminución, en $95 \%$, de los requisitos de capital mínimo para los bancos internacionales, lo que procura atraer hacia Hong Kong nuevas entidades, o a las que abandonaron la plaza en los últimos años (SCMP, 12,12,2001), la controvertida propuesta que autorizaría la venta de participaciones en hedge funds (fondos especulativos) a inversionistas de menudeo (SCMP, 5,11,2001), la desreglamentación de las colocaciones de warrants derivados y la introducción de un instrumento innovador y de gran potencial, las equity-related notes en los mercados financieros (SCMP, 29,11,2001).

En segundo lugar, las autoridades han hecho propuestas a la RPCh para fortalecer los vínculos financieros entre China continental y la plaza de Hong Kong. Así, pidieron al gobierno chino que establezca en Hong Kong -de preferencia a Londres- sus futuras emisiones de títulos de gobierno en US\$ (SCMP , 5,12,2001) y en octubre de 2001 propusieron introducir en el CFI una nueva clase de acciones que se comercializarían en yuanes chinos, con el objetivo de atraer más fondos de la RPCh y de elevar la actividad en los mercados accionarios locales. Simultáneamente, sugirieron que las empresas de Hong Kong podrían cotizar indirectamente en las bolsas de Shanghai y Shenzhen $^{20}$ (SCMP, 17,10,2001). En un mismo orden de ideas, en diciembre del mismo año, la
HKMA pidió a la Comisión Reguladora China de Valores que autorizara la liquidación de las transacciones de acciones "B" 21 en Hong Kong, de preferencia a Nueva York. No cabe duda que la competencia para la preeminencia, en tanto CFI regional, es muy fuerte, y que en el futuro Shanghai podría constituir un competidor serio para parte de las actividades de Hong Kong, perspectiva que pone a las autoridades de la Región Administrativa Especial a mover sus piezas para tomar la delantera. Como lo veremos, Singapur constituye en la actualidad un competidor más peligroso aún: en la segunda parte de este texto, abordaremos el caso de esta ciudad-Estado e intentaremos un análisis de las ventajas respectivas de ambas plazas.

\section{Bibliografía}

Business Week (1999): “Too much of a good thing?", issue $3652,25,10,1999$.

Business Week (2002): "The Black Hole", issue 3768, 04,02,2002.

Cheng, Edward and Raymond Ng (1999): “The Financial Sector and Economic Development in Hong Kong: Implications for Developing Countries", en Masuyama, Seichii, Donna Vandenbrink and Chia Siow Yue, ed. (1999): East Asia's Financial Systems: Evolution and Crisis, Nomura Research Institute and Institute of South East Asian Studies, pp. 200-230.

Carse, David (2000): "The current state of banking reform in Hong Kong”, BIS Review 69, en http://www.bis.org Crawford, Darryl (2001): "Globalization and Guanxi. The Ethos of Hong Kong Finance”, New Political Economy, vol. $6, \mathrm{n}^{\circ} 1$, pp. $45-65$.

FMI (1997): "Hong Kong, China. Growth, Structural Change, and Economic_Stability during the Transition", IMF Occasional Paper, en http://www.imf.org,

FMI (1999): IMF Staff Country Reports núm. 99/34, abril, en http://www.imf.org

FMI (2001): IMF Staff Country Reports núm.1/43, marzo, en http://www.imf.org

FMI (2001): "People's Republic of China-Hong Kong Special Administrative Region: Selected Issues and Statistical Appendix", IMF Country Reports núm. 1/146, agosto, en http://www.imf.org

Goldberg, Manuel (1996): “The Development of a Network of Asia-Pacific Financial Centers", en Das, Dilip ed. (1996):

Emerging Growth Pole. The Asia-Pacific Economy, pp. 380-402.

Hong Kong Monetary Authority: Banking and Supervision, en http://www.info.gov.hk/hkma

Hong Kong Monetary Authority (2002): International Investment Position for 2000 and 2001, en http:// www.info.gov.hk/hkma 
Jao, Y.C. (1997): Hong Kong as an International Financial Centre - Evolution, Prospect and Policies, City University of Hong Kong Press.

Law, CK (1999): Hong Kong Prospects as a Financial Centre, Hong Kong Policy Research Institute, en http:// www.hkpri.org.hk

Lee Sheng Yi (1986): "Developing Asian Financial Centres", en Tan, Augustine \& Basant Kapur, eds. (1986), Pacific Growth and Financial Interdependance, Allen and Unwin, pp. 205-236.

McCaulay, Robert \& Y. K. Mo (1999): "Recent developments in the international banking business of Hong Kong", BIS Quarterly Review, junio, en http://www.bis.org/pub/ rqt9906.pdf, pp. 13-14.

Montes, Manuel (1999): “Tokyo, Hong Kong and Singapore as Competing Financial Centers", en De Brouwer, Gordon \& Wisarn Pupphavesa, eds. (1999), Asia Pacific Financial Deregulation, Routledge, pp. 151-170.

Reed, Howard Curtis (1981): The Preeminence of International Financial Centers, Praeger.

The Economist (1999): "Special Report: Capitals of Capital". The Economist (2002): "Special Report: Rivals more than ever - Hong Kong and Shanghai", vol. 362, issue 8266.

Sassen, Saskia (1999): "Global Financial Centers", Foreign Affairs, vol. 78, núm. 1, enero-febrero, pp. 75-87.

Shi, Joanna (2002): "Hong Kong's External Claims and Liabilities vis-à-vis Mainland China”, en HKMA Quarterly Bulletin, núm. 2, en http.//www.info.gov.hk/hkma/eng/ public/qb200202/fao2.pdf, pp. 15-24.

Yam (1996), en chino, citado por Law, CK (1999).

\section{Diarios}

South China Morning Post; Financial Times (en línea)

\section{Fuentes}

Federación Internacional de Bolsas de Valores, en http:// fibv.com

Fondo Monetario Internacional, Estadísticas Financieras Internacionales.

Hong Kong Monetary Authority, en http:// www.info.gov.hk/hkma

\section{Notas}

1 Se reconocen por lo menos dos etapas en la constitución de una esfera financiera global. La primera, que se da en las décadas de los años 60 y 70, se asocia con el surgimiento del "euromercado", un mercado interbancario internacional desreglamentado, que trata con divisas que no son de curso legal en el país donde toman lugar las operaciones, lo que permite operar al lado de esferas financieras nacionales todavía muy reglamentadas. A partir de inicios de los años 80 se inicia una segunda etapa en la cual los mercados financieros y las instituciones e instrumentos de mercado adquieren un papel preponderante, y las esferas financieras nacionales se liberalizan, cayendo las barreras internas y externas que impedían o limitaban los flujos de capitales.

2 La decisión de atraer la industria de servicios financieros puede obedecer a diversos motivos: por un lado, ciertos países lo hicieron con la finalidad de facilitar la atracción de capitales internacionales y así financiar un crecimiento económico más elevado (Filipinas, Malasia), mientras otros buscaron la contribución que esta industria per se puede hacer al PIB (Singapur).

3 El análisis cubre 50 centros a lo largo de un periodo que va de 1900 hasta 1980.

4 Este criterio diferencia netamente un CFI de un paraíso fiscal, donde prima la ausencia de reglas.

5 Jao (1997) citado por Law (1999) establece una lista más precisa de estas condiciones.

6 Las políticas de represión financiera, conceptualizadas por E. Shaw y R.I. McKinnon en 1973, fueron aplicadas de manera muy común tanto en economías desarrolladas como en países en desarrollo. Incluyen controles de tasas de interés, cajones de crédito obligatorios, topes al crédito, controles de cambio y otros límites a la libertad de los intermediarios.

7 Muchos bancos trasladaron operaciones hacia estas instituciones, mismas que permitieron también la penetración de bancos extranjeros en el mercado, que evadían de esta manera la moratoria de licencias bancarias.

8 En su momento, estas medidas fueron consideradas por numerosos analistas "ortodoxos" como un alejamiento de los principios del "libre-mercado".

9 Al igual que en las crisis anteriores, el colapso se dio a la salida de un ciclo de auge bursátil e inmobiliario.

10 Durante los años 80, la asociación anterior, la Exchange Bank Association, se transformó en Hong Kong Association of Banks.

11 Esta forma legal es preferida en la actualidad a la forma más antigua de sociedad mutualista, y las principales bolsas del planeta la adoptaron o están transformando sus estatutos para hacerlo.

12 Estos títulos comprenden los bills, introducidos en 1990, las notas, creadas en 1993 y las notas a diez años (1996). $\mathrm{Su}$ creación permitió a la HKMA implementar una política monetaria de tipo indirecto.

13 Este sistema ha sido integrado luego (1996) en el sistema de liquidación en bruto en tiempo real (RTGS).

$14 \mathrm{El}$ número de estas instituciones más pequeñas, con sede en Hong Kong, en su gran mayoría, ha venido disminuyendo desde 1981, principalmente en respuesta a cambios en la reglamentación, y posiblemente a procesos de fusiones-adquisiciones.

15 Sin embargo, las instituciones más grandes seguían presentes, con 19 de las primeras 20 a nivel mundial, (Crawford, 2001: 49).

16 Los activos de entidades registradas en el CFI con bancos japoneses caen en una proporción menor, de $56 \%$ (cálculos efectuados por la autora sobre la base de cifras de la HKMA).

17 Este término denota la prima especial de riesgo que pagan las entidades japonesas sobre la tasa base, que 
supera lo pagado por instituciones de países desarrollados.

18 Hasta la quiebra de GITIC, los bancos extranjeros habían dado por descontado que las autoridades chinas garantizarían las deudas contraídas en el extranjero por entidades públicas estatales o regionales y por empresas públicas chinas. Este evento mostró que el gobierno chino no estaba dispuesto a rescatar estas entidades, y significó una elevación en el riesgo percibido por los inversionistas internacionales.

19 Calculado por la autora sobre la base de estadísticas de la HKMA.

20 Esto se daría a través de un instrumento calcado sobre los American Depositary Receipts (ADRs), que permiten que las acciones de empresas extranjeras se comercialicen indirectamente en Estados Unidos, nombrados China Depositary Receipts.

21 Las acciones "B" son acciones de empresas chinas reservadas a inversionistas extranjeros y que cotizan en US \$. T: 